\title{
Efficacy and safety of calcineurin inhibitor treatment for IgA nephropathy: a meta-analysis
}

\author{
Yu-Huan Song ${ }^{1,2}$, Guang-Yan Cai ${ }^{1 *}$, Yue-Fei Xiao², Yi-Ping Wang ${ }^{2}$, Bao-Shi Yuan², Yuan-Yuan Xia', Si-Yang Wang ${ }^{1}$,
} Pu Chen ${ }^{1}$, Shu-Wen Liu ${ }^{1}$ and Xiang-Mei Chen ${ }^{1 *}$

\begin{abstract}
Background: IgA nephropathy is the most common progressive glomerular disease to end stage renal failure worldwide. Calcineurin inhibitors (CNIs) is a selective immunosuppressant widely used in organ transplantation. The efficacy and safety of calcineurin inhibitors for the treatment of IgA nephropathy remain uncertain.

Methods: We performed a systematic literature search using the PubMed, Embase, Science Citation Index, Ovid evidence-based medicine, Chinese Biomedical Literature (CBM) and Chinese science and technology periodicals (CNKI, VIP, and Wan Fang) for randomized, controlled trials of CNIs therapy of IgA nephropathy. Complete remission rate (CR) was defined as proteinuria less than 0.5 or $0.3 \mathrm{~g} / \mathrm{d}$. Partial remission rate (PR) was defined as proteinuria reduced to at least half of the baseline measurement and an absolute value of $>0.5$ or $0.3 \mathrm{~g} / \mathrm{d}$.

Results: Seven relevant trials were conducted with 374 patients enrolled. CNIs plus medium/low-dose steroid had a higher $\mathrm{CR}(\mathrm{RR}=2.51[95 \% \mathrm{Cl}, 1.25$ to 5.04], $P=0.02$ ) compared to therapy with steroid alone or placebo, but were not significant on $\mathrm{PR}(\mathrm{RR}=0.87[95 \% \mathrm{Cl}, 0.32$ to 2.38$] ; P=0.78)$. Also, significant alterations were observed in proteinuria (weighted mean difference, $-0.46 \mathrm{~g} / \mathrm{d},[95 \% \mathrm{Cl}:-0.55$ to -0.24$], P<0.01$ ) with no differences were found in serum creatinine ( $\mathrm{SCr}$ ) (weighted mean difference, $0.57,95 \% \mathrm{Cl}:-4.05$ to $5.19 ; \mathrm{P}=0.78$ ) and estimated glomerular filtration rate (eGFR) (weighted mean difference, 1.13,95\% Cl:-4.05 to 6.32; $P=0.34$ ) level between the two groups. $\mathrm{CNI}$ therapy was associated with an increased risk for adverse events ( $\mathrm{RR}=2.21,95 \% \mathrm{Cl}: 1.52$ to $3.21, P<0.01$ ), such as gastrointestinal and neurological symptoms or hirsutism.
\end{abstract}

Conclusions: CNIs might provide renal protection in patients with IgAN, but at an increased risk of adverse events. Reliably defining the efficacy and safety of CNIs in IgAN requires a high-quality trial with a large sample size.

Keywords: IgA nephropathy, Calcineurin inhibitor, Cyclosporine A, Tacrolimus

\section{Background}

IgA nephropathy is the most common primary glomerular disease worldwide. A wide variety of treatments have attempted to reduce kidney burden and the high risk of kidney failure events in this population. IgAN is an autoimmune kidney disease, indicating that immunosuppressive therapy may be helpful. Immunosuppressive therapy is supposed to reduce the deterioration in kidney function

\footnotetext{
* Correspondence: caiguangyan@sina.com; xmchen301@126.com

'Department of Nephrology, Chinese PLA General Hospital, Chinese PLA Institute of Nephrology, State Key Laboratory of Kidney Diseases, National Clinical Research Center for Kidney Diseases, 28 Fuxing Road, Beijing 100853, People's Republic of China

Full list of author information is available at the end of the article
}

as well as a reduction in proteinuria. The core I $\beta 3-G a l-T$ specific molecular chaperone (Cosmc) gene expression was decreased in IgAN patients. Immunosuppressive therapy can up-regulate the Cosmc expression in peripheral lymphocytes of IgAN patients. It might be the underlying mechanism of immunosuppressive therapy used in treating IgAN $[1,2]$. It has been proven that calcineurin inhibitors (CNIs) which include cyclosporine A (CsA) and tacrolimus (TAC), can suppress the immune response by downregulating the transcription of various genes in $\mathrm{T}$ cells.

There are only a few small studies available using CNIs for the treatment of IgAN ten years ago [3], mainly 
affected by the very first report that discouraged the use of this medication in IgAN due to an increase in serum creatinine ( $\mathrm{SCr}$ ), although the complication was reversible [4]. From then on, due to the lack of controlled clinical trials, the benefit and risk of CNIs in the treatment of IgAN remained uncertain [5-8]. Recently, several randomized controlled trials (RCTs) suggested that CNIs might be effective for IgAN. Moreover, there are a few other studies that have successfully used CNIs in resistant IgAN patients, which demonstrated that CNIs could decrease proteinuria in IgAN patients who showed resistance to steroids and/or other immunosuppressants [9]. We therefore conducted this meta-analysis of all available RCTs to comprehensively ascertain the benefits and risks of $\mathrm{CNI}$ treatment in comparison with steroids or placebos in patients with IgAN.

\section{Methods}

\section{Identification of eligible studies}

Two researchers (GYC and YHS) performed a systematic literature search using the PubMed, Embase, Science Citation Index, Ovid evidence-based medicine, Chinese Biomedical Literature (CBM) and Chinese science and technology periodicals (CNKI, VIP, and Wan Fang) databases without any language restriction. All of the relevant studies were published between 1986 and July 2016. The following key words and subject terms were used in the search: 'IgA nephropathy', 'immunoglobulin A nephropathy', 'IgA nephritis, 'IgA glomerulonephritis', 'Berger's disease', 'cyclosporine A', 'CsA', 'tacrolimus', 'FK506', and their derivative words.

\section{Inclusion and exclusion criteria}

Two authors independently selected information from the studies and disagreement was resolved by consensus. The titles and abstracts were scanned to exclude any trials that were clearly irrelevant in the first stage. The full texts of the relevant articles were read in order to determine whether they contained information on the topic of interest in the second stage. The baseline data of patients, proteinuria level, doses and duration of CNIs use, follow-up duration, clinical parameters and adverse events were included in the extracted information.

Inclusion criteria consisted of: (1) the study design was a RCT; (2) the study focused on patients with biopsyproven IgA nephropathy; (3) the study compared TAC or CsA with corticosteroid or placebo in the induction therapy of IgAN; and (4) at least one of the following outcomes was reported: the complete remission (CR) or partial remission (PR) of proteinuria, changes of clinical outcomes (including proteinuria, serum creatinine or eGFR) and adverse events.

$\mathrm{CR}$ was defined as proteinuria less than 0.5 or $0.3 \mathrm{~g} / \mathrm{d}$ and a normal serum creatinine (Scr) level. PR are among those patients who did not have a CR, was defined as proteinuria reduced to at least half of the baseline measurement and an absolute value of $>0.5$ or $0.3 \mathrm{~g} / \mathrm{d}$ and as well as a relatively stable Scr level (variation less than 25\%).

Exclusion criteria were: (1) did not including English abstract; (2) studies including minors; (3) did not describe the numbers of patients who recovered, deteriorated, or had renal replacement treatment clearly.

\section{Assessment of trial quality}

We assessed the quality of RCTs using a standard scoring system proposed in the Jadad scale criteria [10]. These included: (1) whether the randomization method was appropriately performed; (2) whether double-blindness was used in the RCT and whether it was appropriate; (3) whether the report (the patient number and reasons) of withdrawal and drop-outs was stated clearly. We classified the RCTs as high quality if they scored $>2$. Otherwise, assessed them as low quality [11].

\section{Statistical analysis}

Cochrane RevMan 5.3 was used to perform statistical analyses. The results were stated as relative risks (RR), for dichotomous outcomes, and weighted mean differences, for continuous outcomes, with 95\% confidence intervals $(95 \% \mathrm{CI})$. The heterogeneity Q statistic test was used to analyze heterogeneity among the included trials. If it indicated heterogeneity $(p<0.05)$ across trials, the DerSimonian and Laird method in the random effect model was selected. Otherwise, the Mantel-Haenszel method in the fixed-effect model was used.

\section{Results}

\section{Studies included in the meta-analysis}

The comprehensive literature retrieval yielded 1156 articles. Of these, 71 were acquired in full-text form. Seven RCTs were identified as appropriate for inclusion in this meta-analysis (Fig. 1). The included studies provided information on a total of 374 patients. Table 1 showed the summarized characteristics of the included studies. Among them, Six studies (334 patients) included IgAN patients with proteinuria $1-3.5 \mathrm{~g} / \mathrm{d}[4,12-16]$. One study (40 patients) included patients mainly with mild or moderate proteinuria [17]. The Jadad method was assessed for the quality of the RCTs. It ranged from 2 to 7 and two trials were of high quality (Jadad score $=7$ ).

\section{Trial outcomes}

\section{Effect on Proteinuria}

Five studies assessed CR or PR in a total of 225 patients, CR ( $R R=2.51 ; 95 \%$ CI 1.25 to 5.04) occurred more frequently among people in the CNIs group compared with the control group. PR did not reach a significant 


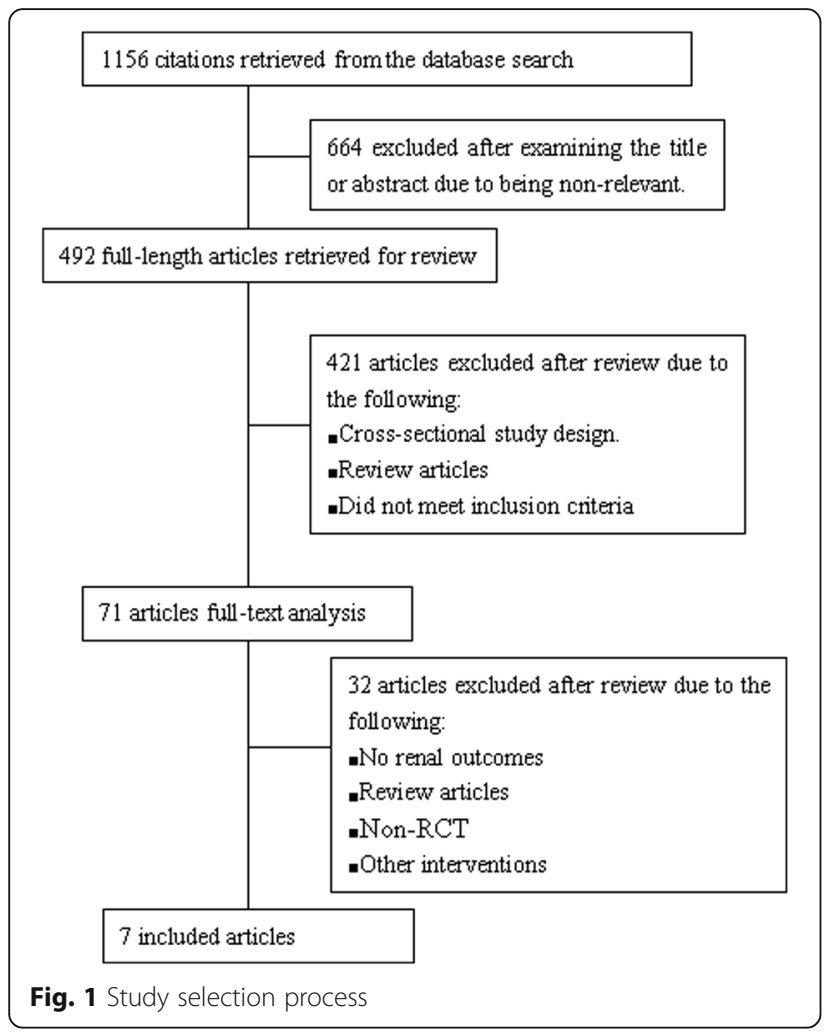

difference between CNIs and steroid alone or Placebo ( $\mathrm{RR}=0.87,95 \%$ CI 0.32 to 2.38$)$ (Fig. 2).

Three studies assessed urinary protein excretion in a total of 169 patients. 24-h proteinuria was significantly lower in patients using CNIs than in the control groups (weighted mean difference, $-0.46 \mathrm{~g} / \mathrm{d}, 95 \% \mathrm{CI}:-0.55$ to $-0.24)$ at the end of treatment or during follow-up (Fig. 3). The randomized effects model was selected because heterogeneity was significant $(P<0.01)$.

\section{Effect on Scr and eGFR}

There were five studies assessed eGFR or Scr in patients. Both eGFR (weighted mean difference, 1.13,95\% CI:-4.05 to $6.32 P=0.34$ ) (Fig. 4) and Scr (weighted mean difference, $0.57,95 \% \mathrm{CI}:-4.05$ to $5.19, P=0.78$ ) (Fig. 5) were not significantly lower in patients using CNIs than in the control groups at the end of treatment or during follow-up. The fixed effects model was used because heterogeneity was not significant $(P=0.78)$.

\section{Adverse events}

All adverse events were collected which mentioned in the included articles, and the most prevalent events were analyzed in the synthesis. The following outcomes were included: liver function disorder, respiratory symptoms, such as infection, elevated blood sugar, cardiovascular symptoms, such as hypertension, eyesight degradation, hirsutism, gingivitis, musculoskeletal symptoms, gastrointestinal discomfort, neurologic discomforts, hematologic symptoms, drop in eGFR, urinary tract infection, and withdrawal. Data on adverse events potentially caused by treatment were collected from the RCTs (Fig. 6).

CNIs therapy was associated with an increased risk for several events (RR, 2.21 [95\% CI, 1.52 to 3.21]). Significantly, patients receiving CNIs appeared to have a higher risk of experiencing gastrointestinal discomfort or liver function disorder (RR, 28.89 [95\% CI, 5.35 to 155.96]), neurologic or musculoskeletal symptoms (RR, 9.80 [95\% CI, 2.50 to 38.36$]$ ), and hirsutism or gingivitis (RR, 11.65 [95\% CI, 2.68 to 50.61]). However, fewer patients who received CNIs developed elevated blood sugar (RR, 0.36 [95\% CI, 0.14 to 0.91]). Hypertension and drop in eGFR of more than $25 \%$ from the baseline did not reach a significant difference between CNIs and steroid alone or placebo. The fixed-effects model was selected because heterogeneity was undetectable when the effect sizes of side effects were evaluated $(P>0.05)$.

In 7 RCTs, there were five patients in $\mathrm{CNI}$ groups and one patient in control group who withdraw from the therapy. One patient showed irreversible kidney failure even with a dosage reduction of CsA and treatment was subsequently stopped [4]. Three patients in the CsA group and one patient in the control group developed severe pneumonia in the second to third month of treatment, after which the steroid and CsA therapies were discontinued [15]. One patient was advised to discontinue the TAC after the fourth week of treatment because of general weakness and myalgia [17].

\section{Publication bias}

The funnel plots exhibited symmetric patterns for both proteinuria and renal function, as shown in Figs. 7 and 8. Because the sample sizes of the 7 RCTs included in this meta-analysis were all small, we conducted Begg's test to evaluate the publication bias using Stata software, which indicated no significant heterogeneity in the 7 RCTs.

\section{Discussion}

IgAN is the most common type of glomerulonephritis worldwide $[18,19]$. It is now known to slowly progress to end-stage renal disease (ESRD) [20-23]. Proteinuria is one of the strongest independent prognostic factors $[24,25]$. IgAN with severe proteinuria are conventionally subjected to treatment with various immunosuppressive regimens with conflicting results $[26,27]$. Studies showed that immunosuppressive therapy for IgAN may reduce the risk of ESRD by 70\% compared with supportive therapy after > 5-year follow-up [28, 29]. CNIs are widely used as immunosuppressive drugs. Studies suggest that CNIs are effective in decreasing proteinuria in a variety of glomerular diseases, including IgAN $[30,31]$. 


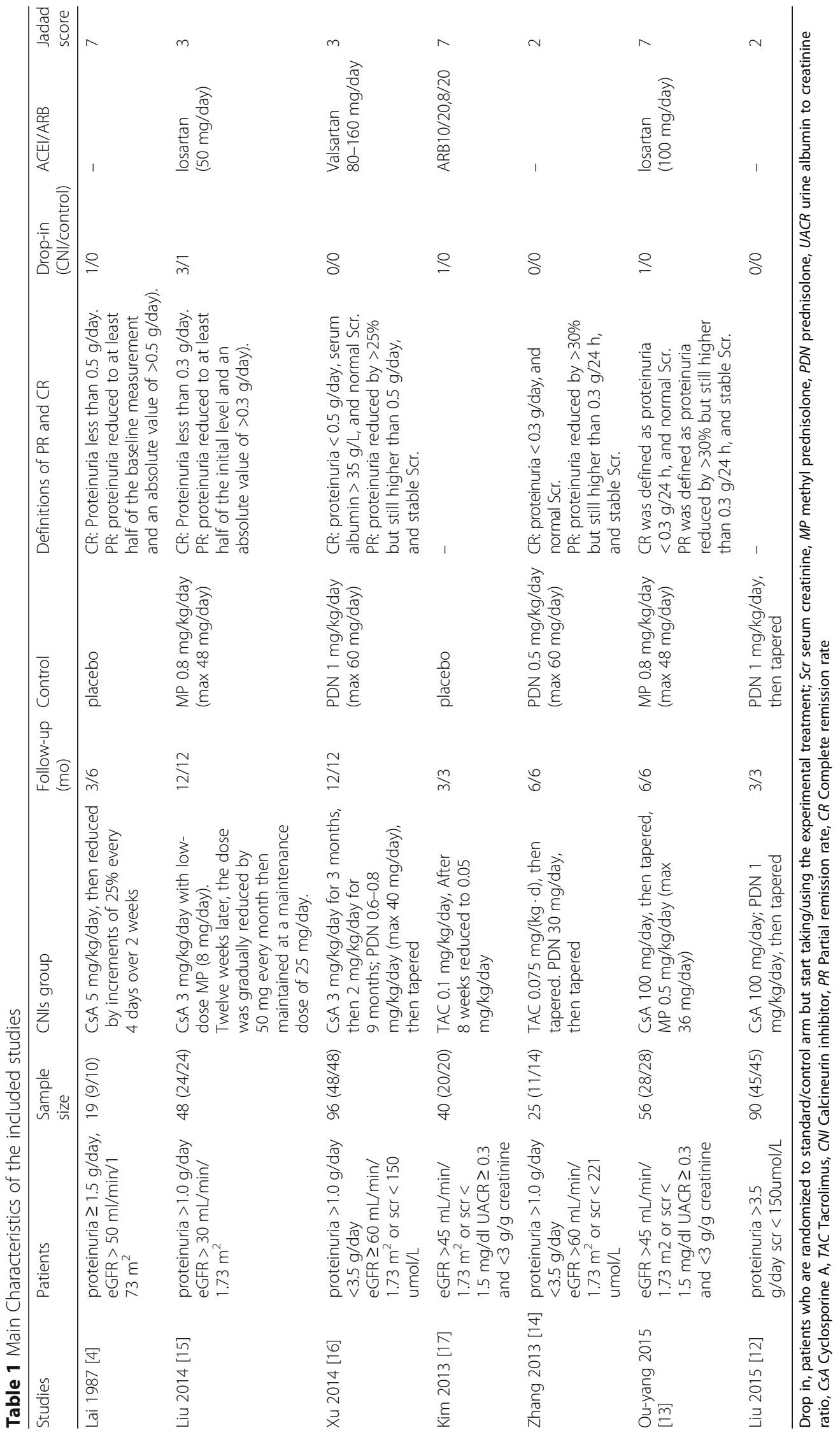




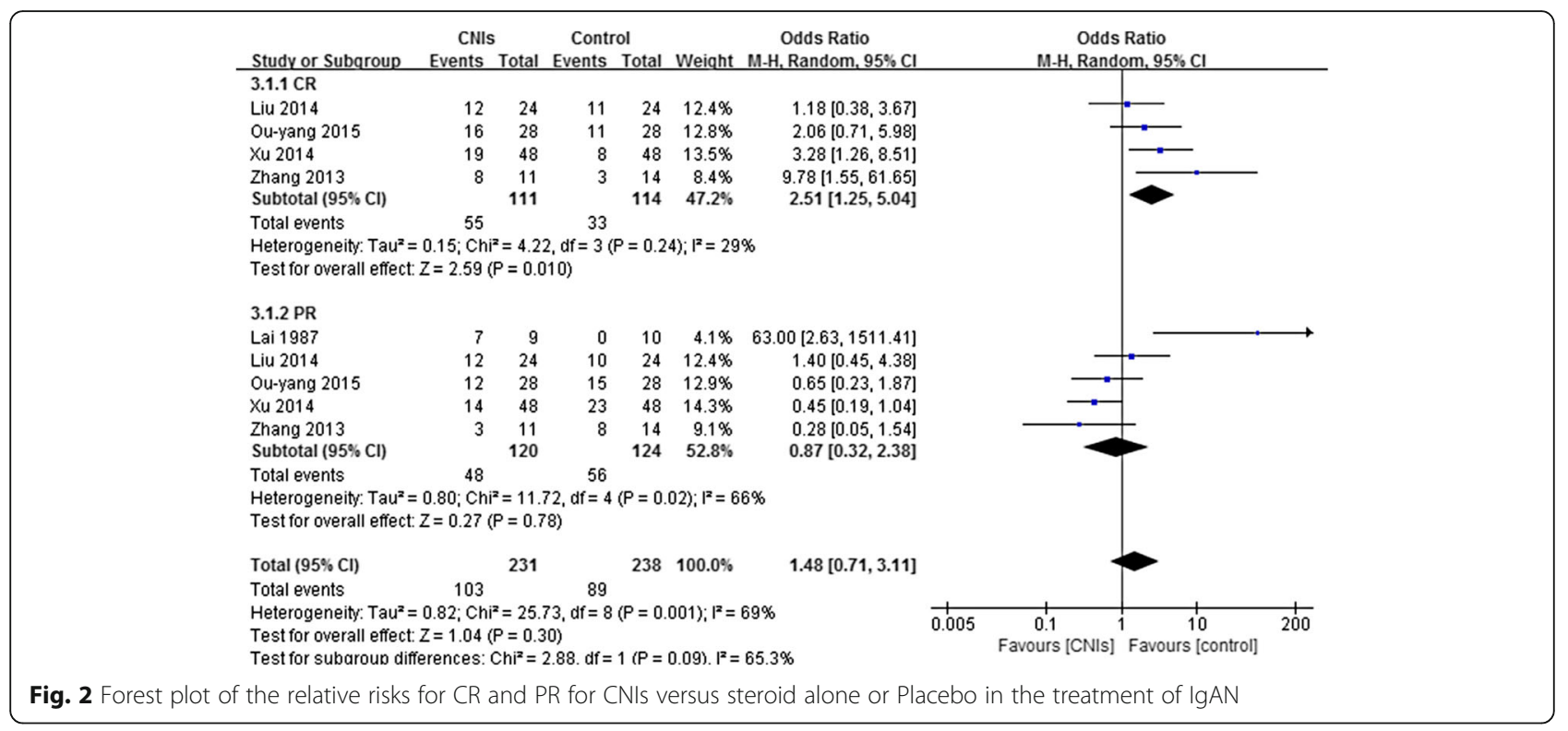

So far, few RCTs have analyzed the role of CNIs in patients with IgAN. The current meta-analysis of seven trials involving 374 patients with IgAN revealed that the combination of CNIs and medium/low-dose steroid was more effective in reducing proteinuria compared with the steroid group alone, suggesting a synergistic effect between CNIs and steroid. Similar to our findings, several studies also indicated that patients with IgAN could experience significant improvement in proteinuria and hypoalbuminemia during CNI treatment [32]. In addition, the risk of developing elevated blood sugar appeared lower in patients treated with CNIs in comparison with placebo or steroid. Moreover, this meta-analysis concluded that there was no significant difference in the risk of renal impairment or rate of decline of eGFR between two groups.

CNIs were associated with a higher incidence of experiencing gastrointestinal discomfort or liver function disorder, and neurological or musculoskeletal symptoms than placebo or steroid. They were also associated with a higher incidence of experiencing hirsutism or gingivitis. This was consistent with the results of studies containing CNIs [33].
IgA patients who achieved remission had far better outcomes than those who never achieved remission $[34,35]$. These findings suggest that achieving remission, whether $\mathrm{CR}$ or $\mathrm{PR}$, is important in IgA patients to improve renal survival, irrespective of glomerular disease type. In current systematic review, CNIs group increased the rates of $\mathrm{CR}$ compared with steroid alone or placebo.

There are a few other studies that have successfully used CsA or TAC in resistant IgAN patients. In one retrospective case series of 13 adult patients with IgAN and significant proteinuria, more than half of the patients did respond to CsA therapy with or without steroids, with long-term remission. A rise in Scr was observed in only two patients, and was mild and reversible in these cases [36]. In a non-randomized study, Chabova and colleagues administered $5 \mathrm{mg} / \mathrm{kg} /$ day of CsA plus alternate day 5-10 $\mathrm{mg}$ prednisolone to 6 IgAN patients with nephrotic-range proteinuria and normal Scr, who were resistant to three months of glucocorticoid therapy [37]. They aimed for a trough serum cyclosporine level of $70-150 \mathrm{ng} / \mathrm{mL}$ and continued the regimen for one year. After one month of treatment, proteinuria reduced from $4.66 \pm 0.43 \mathrm{~g} /$ day to $1.38 \pm 0.29 \mathrm{~g} /$ day, and after one

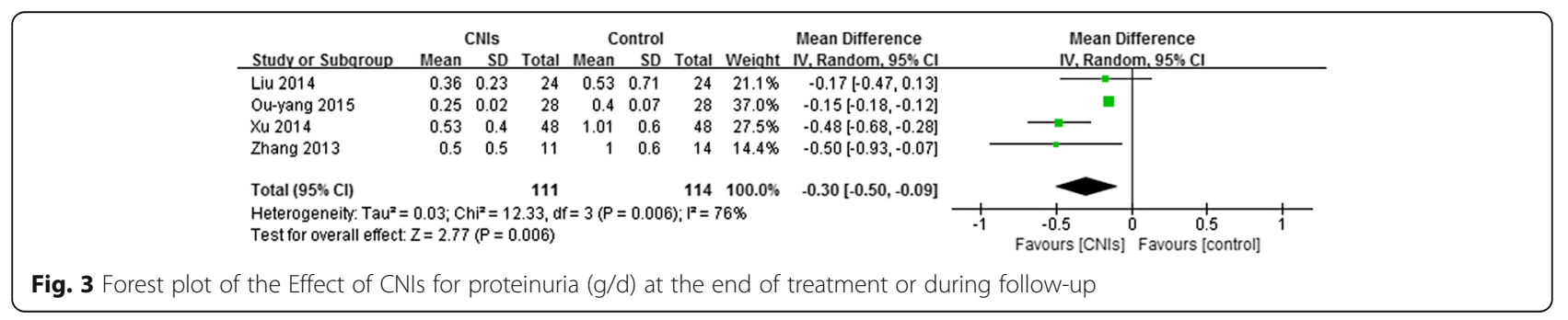




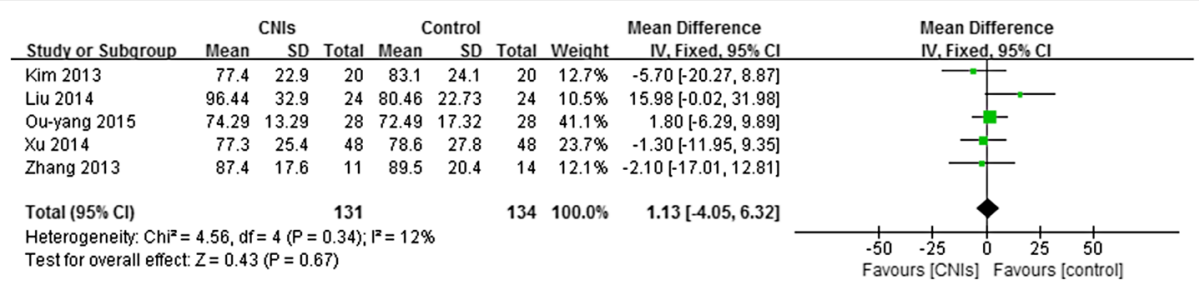

Fig. 4 Forest plot of the Effect of CNIs on eGFR at the end of treatment or during follow-up

year to $0.59 \pm 0.14 \mathrm{~g} /$ day. GFR did not differ significantly from the baseline in two years. In a retrospective study by Shin and colleagues on 14 children with IgAN, a significant decrease in proteinuria and increase in serum albumin concentration without any rise in Scr level was observed [38]. A decrease in histologic grade of IgAN was seen in a follow-up biopsy of $50 \%$ of the patients. These researchers suggested that CsA has a significant role in decreasing proteinuria and reversing kidney pathology in children with IgAN. In another interesting recent study, remission of nephrotic-range proteinuria could be induced in 9 of 11 IgAN patients with the use of TAC, which was explained through the effect of the drug in podocyte cytoskeleton stabilization through inhibition of calcineurin expression [39].

However, there is still a strong debate regarding the use of CNIs, especially CsA for the treatment of proteinuria in IgAN, mainly due to concerns about the possible increase in Scr caused by CsA, although it is being used as one of the main immunosuppressive agents in various other proteinuric glomerulonephritides. It concluded that there was no significant difference in the risk of ESRD or rate of decline of GFR in patients treated with CsA or placebo in a meta-analysis.

Our meta-analysis had four limitations. First, the proteinuria outcomes were measured while on CNI, whether a reduction in proteinuria while on a CNI will be sustained or will rebound after the CNI is stopped is not certain. We should also address the limitation of using proteinuria as a surrogate outcome measure, and the implication of rebounding proteinuria after stopping CNI. Second, the renal outcomes that were assessed were over likely too short a time period to see any beneficial or detrimental effects from chronic CNI use. Our meta-analysis do not show significant benefit on kidney function, as serum creatinine or eGFR. Longterm, large sample, multicenter RCTs are needed to confirm the efficacy and safety of CNIs in the treatment of IgAN. Third, the number of subjects included in this analysis was not particularly great. Finally, there appears to be lack of published small studies with negative outcomes. The risk of publication bias in which studies with negative results is also a limitation.

The current meta-analysis was generally consistent with these reviews $[7,8]$. Thus, we believe that the results of our studies can help to prevent the discouragement of the use of this medication for an idiopathic immunologic disease without many therapeutic choices. The fear of increase in Scr seems to have prevented the researchers from designing clinical trials to study this valuable immunosuppressive agent in the treatment of IgAN, and we suggest starting such trials for a better long-term judgment.

\section{Conclusions}

Prescription of CNI combined with medium-dose steroid resulted in significant reduction in proteinuria without deteriorated renal function, showing that CNI may be a promising agent for IgAN. It is important to use the lowest effective dosage of CNIs and monitor its level closely because of possible complications. Larger RCTs of CNIs which are sufficiently powered to evaluate patient-relevant end points, including adverse events, and that examine the optimal duration of treatment are now required in IgAN patients with a range of kidney function.

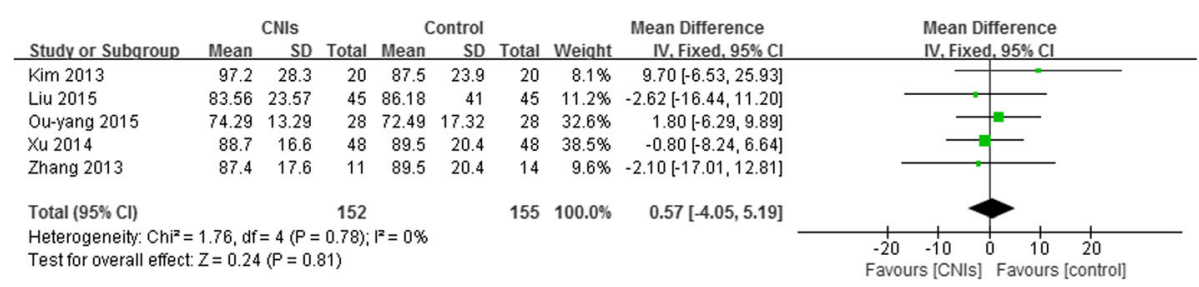

Fig. 5 Forest plot of the Effect of CNIs on $\mathrm{SCr}$ at the end of treatment or during follow-up 


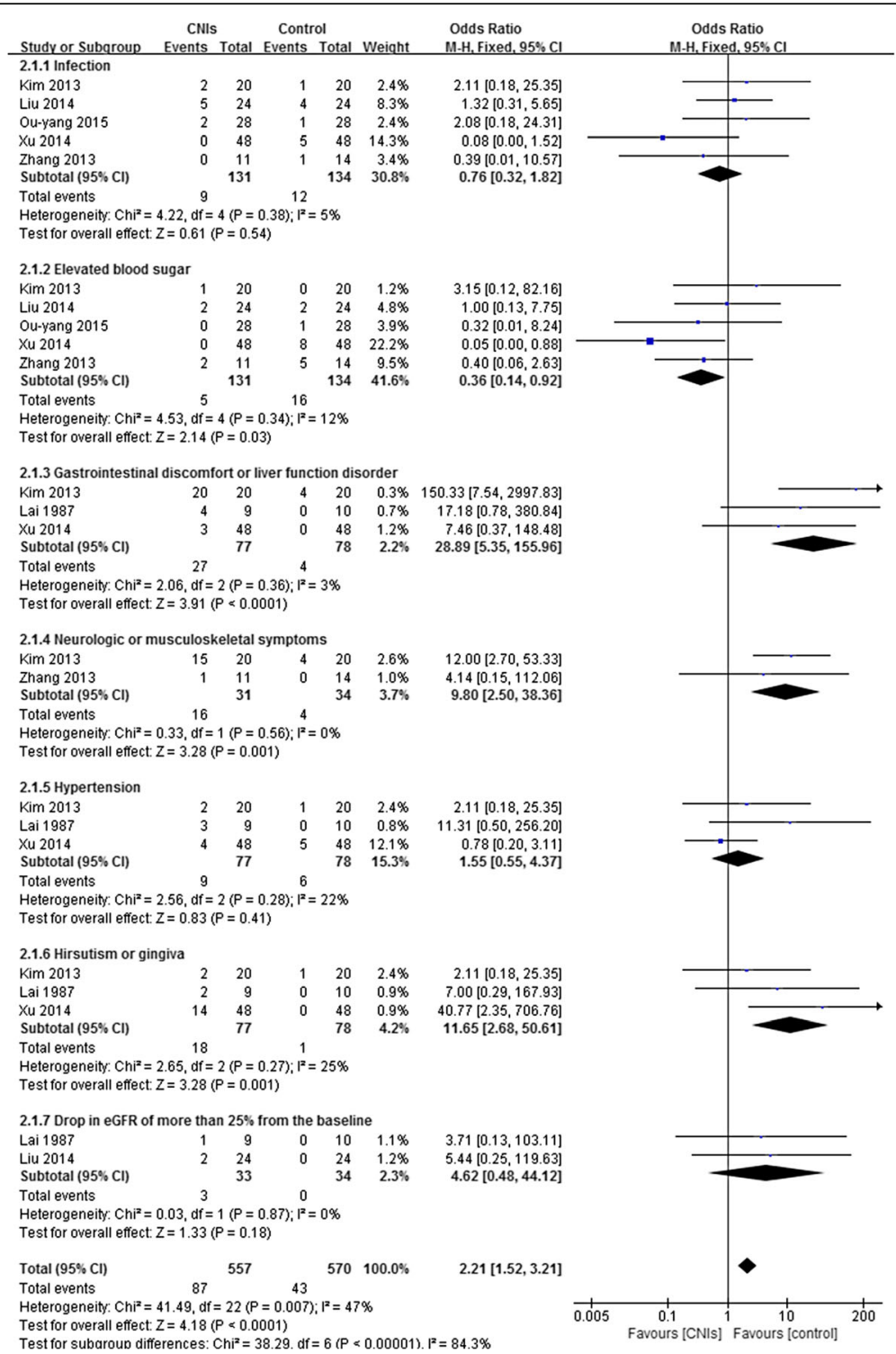

Fig. 6 Forest plot of the relative risks for adverse events at the end of treatment or during follow-up

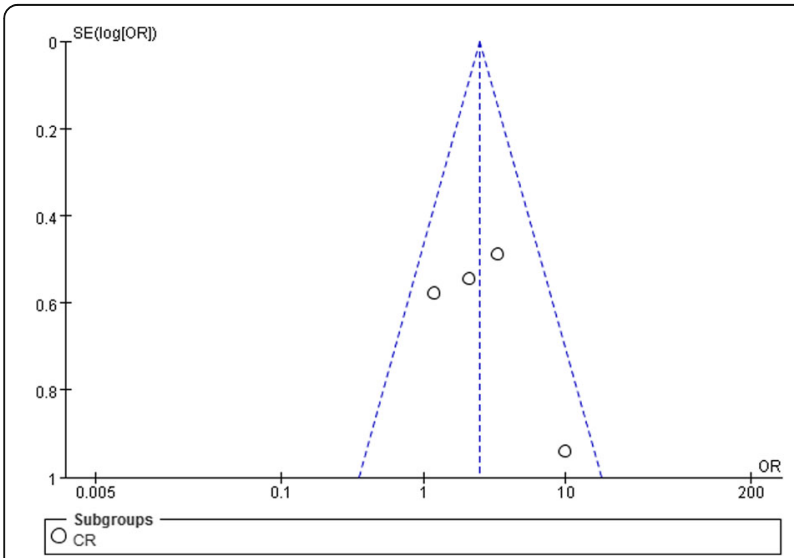

Fig. 7 Funnel plot of four RCTs for Effect in proteinuria CR of CNIs treatment of IgAN patients

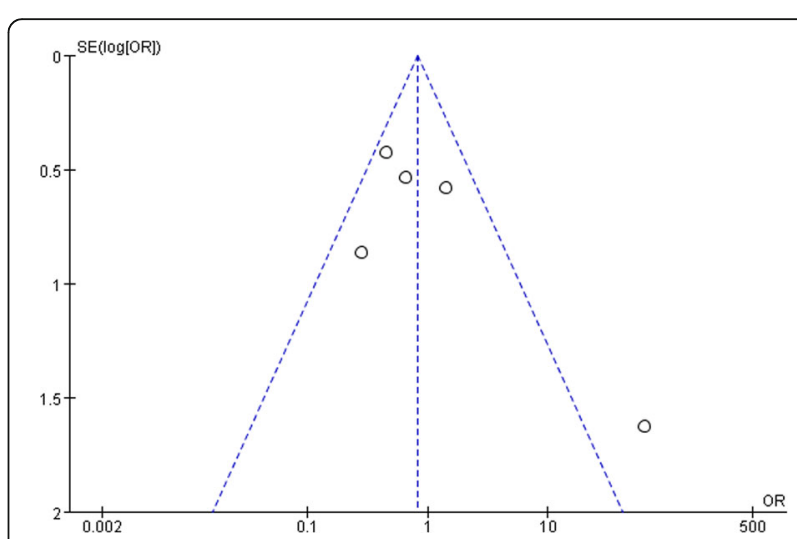

Fig. 8 Funnel plot of five RCTs for Effect in SCr of CNIs treatment of IgAN patients 


\section{Abbreviations}

95\% Cl: 95\% confidence intervals; CNIs: Calcineurin inhibitors; Cosmc: Core I $\beta 3$-Gal-T-specific molecular chaperone; CR: Complete remission; CSA: Cyclosporine A; ESRD: End-stage renal disease; IgAN: Immunoglobulin A nephropathy; PR: Partial remission; RCT: Randomized controlled trial; RR: Relative risks; Scr: Serum creatinine; TAC: Tacrolimus

\section{Acknowledgments}

We thank all of the investigators who responded to our inquiries regarding their original studies. This study was supported by 863 program (2012AA02A512), Twelfth Five-Year Plan (2011BAl10B03, 2013BAl09B05, 2015BAl12B06), 973 program (2013CB530800), and the NSFC (81171645). There are no conflicts of interest to declare.

\section{Funding}

This work was supported by the National Key Technology Research and Development Program (2015BAl12B06, 2013BAI09B05), 973 program (2013CB530800), the 863 program (2012AA02A512), and the NSFC (81171645).

\section{Availability of data and materials}

All the data supporting the conclusions of this article are contained within the manuscript. The individual patient-level dataset was not made publically available due to containing potentially identifying patient data; however, the study dataset may be made available from the authors upon request.

\section{Authors' contributions}

Each author contributed to the conception and design of the study and interpretation of the data. YHS conceived and designed the study, participated in the literature searches and drafted the manuscript. GYC participated in the design of the study and the literature search. YHS drafted the article and all authors revised it critically for important intellectual content. All authors gave final approval of the version to be published. Specific contributions are as follows: Study concept and design: YHS, GYC. Acquisition of data: YHS and GYC. Data Analysis: BSY and YHS. Interpretation of the data: YHS, GYC, YFX, YPW, YYX. Drafting of the manuscript: YHS and GYC. Critical revision of the manuscript for important intellectual content: YHS, GYC, SYW, SWL, PC and XMC.

\section{Competing interests}

The authors declare that they have no competing interests.

\section{Consent for publication}

Not applicable.

\section{Ethics approval and consent to participate}

This study protocol does not need ethics committee approval.

\section{Author details}

'Department of Nephrology, Chinese PLA General Hospital, Chinese PLA Institute of Nephrology, State Key Laboratory of Kidney Diseases, National Clinical Research Center for Kidney Diseases, 28 Fuxing Road, Beijing 100853, People's Republic of China. ${ }^{2}$ Department of Nephrology, Aerospace Central Hospital, Beijing, China.

Received: 12 August 2016 Accepted: 31 January 2017

\section{Published online: 13 February 2017}

\section{References}

1. Xie L, Tan C, Fan J, et al. Mycophenolic acid reverses lgA1 aberrant glycosylation through up-regulating Cosmc expression in IgA nephropathy. Int Urol Nephrol. 2013:45(2):571-9.

2. Wei $Q$, Xiang Z, Jun MF, et al. External suppression causes the low expression of the Cosmc gene in IgA nephropathy. Nephrol Dial Transplant. 2008:23(5):1608-14.

3. Merlin T. Specific management of IgA nephropathy: role of cyclosporin and other therapies. Prevention of Progression of Kidney Disease (The CARI Guidelines) 2005; Sept:S149-153.

4. Lai KN, Lai FM, Li PK, et al. Cyclosporin treatment of IgA nephropathy: a short term controlled trial. Br Med J (Clin Res Ed). 1987;295(6607):1165-8.
5. Cattran DC. Current status of cyclosporin A in the treatment of membranous, IgA and membrano proliferative glomerulonephritis. Clin Nephrol. 1991;35 Suppl 1:S43-7.

6. Nolin L, Courteau M. Management of IgA nephropathy: Evidence-based recommendations. Kidney Int Suppl. 1999;70:S56-62.

7. 7 Thomas M: Specific Management of IgA Nephropathy: Role of Cyclosporin and Other Therapies. The CARI Guidelines - Caring for Australasians with Renal Impairment Prevention of Progression of Kidney Disease 2006; April: S149-153.

8. Samuels JA, Strippoli GF, Craig JC, et al. Immunosuppressive treatments for immunoglobulin A nephropathy: A meta-analysis of randomized controlled trials. Nephrology (Carlton). 2004;9:177-85.

9. Fan L, Liu Q, Liao Y, et al. Tacrolimus is an alternative therapy option for the treatment of adult steroid-resistant nephrotic syndrome: a prospective, multicenter clinical trial. Int Urol Nephrol. 2013:45(2):459-68.

10. Jadad AR, Moore RA, Carroll D, et al. Assessing the quality of reports of randomized clinical trials: is blinding necessary? Control Clin Trials. 1996;17:1-12.

11. Moher D, Pham B, Jones A, et al. Does quality of reports of randomised trials affect estimates of intervention efficacy reported in meta-analyses? Lancet. 1998:352(9128):609-13.

12. Liu JJ, Huang YH, Peng YM, et al. Effect of cyclosporine combined with glucocorticoids on $\lg \mathrm{A}, \mathrm{C} 3$ and $\lg \mathrm{A} / \mathrm{C} 3$ in patients with $\lg \mathrm{A}$ nephropathy. Prog Modern Biomed. 2015;15(25):4885-91.

13. Ouyang HY. Measuring inconsistency in meta-analyses. Chin J Chin Pharmacol. 2015;31(18):1825-7.

14. Zhang JX, Qian JZ. Clinical efficacy of tacrolimus combined with small does of hormone therapy on IgA nephropathy. J Jiangsu University. 2013;23(3):263-7.

15. Liu H, Xu X, Fang Y, et al. Comparison of glucocorticoids alone and combined with cyclosporine a in patients with IgA nephropathy: a prospective randomized controlled trial. Intern Med. 2014;53:675-81.

16. Xu L, Liu ZC, Guan GJ, et al. Cyclosporine A combined with medium/low doseprednisone in progressive IgA nephropathy. Kaohsiung Journal of Medical Sciences. 2014;30:390-5.

17. Kim YC, Chin HJ, Koo HS, et al. Tacrolimus Decreases Albuminuria in Patients with IgAN ephropathy and Normal Blood Pressure: A Double-Blind Randomized Controlled Trial of Efficacy of Tacrolimus on IgA Nephropathy. PLoS One. 2013;8(8):e71545.

18. Peters $\mathrm{HP}$, van den Brand JA, Berger SP, et al. Immunosuppressive therapy in patients with IgA nephropathy. Neth J Med. 2015;73(6):284-9.

19. Kiryluk K, Novak J, Gharavi AG. Pathogenesis of immunoglobulin a nephropathy: recent insight from genetic studies. Annu Rev Med. 2013;64:339-56.

20. Beukhof JR, Kardaun O, Schaafsma W, et al. Toward individual prognosis of IgA nephropathy. Kidney Int. 1986;29(2):549-56.

21. D'Amico G. Natural history of idiopathic IgA nephropathy: role of clinical and histological prognostic factors. Am J Kidney Dis. 2000;36(2):227-37.

22. Koyama A, Igarashi $M$, Kobayashi M. Natural history and risk factors for immunoglobulin A nephropathy in Japan. Research Group on Progressive Renal Diseases. Am J Kidney Dis. 1997;29(4):526-32.

23. Nicholls KM, Fairley KF, Dowling JP, et al. The clinical course of mesangial IgA associated nephropathy in adults. Q J Med. 1984;53(210):227-50.

24. Cai GY, Chen XM. Immunoglobulin A nephropathy in China: progress and challenges. Am J Nephrol. 2009:30(3):268-73.

25. Bartosik LP, Lajoie G, Sugar L, et al. Predicting progression in IgA nephropathy. Am J Kidney Dis. 2001;38(4):728-35.

26. Strippoli GF, Manno C, Schena FP. An "evidence-based" survey of therapeutic optionsfor IgA nephropathy: assessment and criticism. Am J Kidney Dis. 2003;41:1129-39.

27. Lv J, Xu D, Perkovic V, et al. Corticosteroid therapy in IgA nephropathy. J Am Soc Nephrol. 2012;23:1108-16.

28. Tian $L$, Shao $X$, Xie $Y$, Wang $L$, et al. The long-term efficacy and safety of immunosuppressive therapy on the progression of IgA nephropathy: a meta-analysis of controlled clinical trials with more than 5-year follow-up. Expert Opin Pharmacother. 2015;16(8):1137-47.

29. Vecchio M, Bonerba B, Palmer SC, et al. Immunosuppressive agents for treating IgA nephropathy. Cochrane Database Syst Rev. 2015;3(8):CD003965.

30. Arikan H, Koc M, Cakalagaoglu F, et al. Tacrolimus rescue therapy in resistant or relapsing cases of primary glomerulonephritis. J Nephrol. 2008;21(5):713-21. 
31. Machiguchi T, Tei M, Ono T, et al. Re-biopsy in a patient with IgA nephropathy showing success of treatment with cyclosporin A and angiotensin-II receptor blocker. Clin Exp Nephrol. 2002;6(3):166-9.

32. Wei $P, Y i$ T, Zheng J, et al. The effect of calcineurin inhibitors in the treatment of IgA nephropathy:A systematic review and meta-analysis (PRISMA). Medicine (Baltimore). 2016;95(35):e4731.

33. Shin DH, Lee MJ, Oh HJ, et al. Stepwise treatment using corticosteroids alone and in combination with cyclosporine in korean patients with idiopathic membranous nephropathy. Yonsei Med J. 2013;54(4):973-82.

34. Sandsmark DK, Messé SR, Zhang X, et al. Proteinuria, but Not eGFR, predicts stroke risk in chronic kidney disease: chronic renal insufficiency cohort study. Stroke. 2015;46(8):2075-80

35. Kim JK, Kim JH, Lee SC, et al. Clinical features and outcomes of IgA nephropathy with nephrotic syndrome. Clin J Am Soc Nephrol. 2012:7(3):427-36.

36. Ossareh $S$, Madadi B, Joodat R. Effect of cyclosporine a in the treatment of proteinuric patients with immunoglobulin A nephropathy. Saudi I Kidney Dis Transpl. 2014;25(3):661-6.

37. Chábová V, Tesar V, Zabka J, et al. Long term treatment of IgA nephropathy with CycloporineA. Ren Fail. 2000;22:55-62.

38. Shin JI, Lim BJ, Kim PK, et al. Effects of cyclosporine A therapy combined with steroids and angiotensin converting enzyme inhibitors on childhood IgA nephropathy. J Korean Med Sci. 2010;25:723-7.

39. Zhang Q, Shi SF, Zhu L, et al. Tacrolimus improves the proteinuria remission in patients with refractory IgA nephropathy. Am J Nephrol. 2012;35:312-20.

\section{Submit your next manuscript to BioMed Central and we will help you at every step:}

- We accept pre-submission inquiries

- Our selector tool helps you to find the most relevant journal

- We provide round the clock customer support

- Convenient online submission

- Thorough peer review

- Inclusion in PubMed and all major indexing services

- Maximum visibility for your research

Submit your manuscript at www.biomedcentral.com/submit

) Biomed Central 